Homology, Homotopy and Applications, vol.15(2), 2013, pp.281-300

\title{
n-RELATIVE CATEGORIES: A MODEL FOR THE HOMOTOPY THEORY OF $n$-FOLD HOMOTOPY THEORIES
}

\author{
C. BARWICK AND D. M. KAN
}

(communicated by Daniel Dugger)

\begin{abstract}
We introduce, for every integer $n \geqslant 1$, the notion of an $n$ relative category and show that the category of the small $n$ relative categories is a model for the homotopy theory of $n$-fold homotopy theories, i.e., homotopy theories of ... of homotopy theories.
\end{abstract}

\section{Background and motivation}

In this introduction we

- recall some results of (higher) homotopy theory, and

- explain how they led to the current manuscript.

We start with

\subsection{Rezk and re-Rezk}

In [R] Charles Rezk constructed a left Bousfield localization of the Reedy structure on the category s $\boldsymbol{S}$ of small simplicial spaces (i.e., bisimplicial sets) and showed it to be a model for the homotopy theory of homotopy theories.

Furthermore it was noted in [B] (and a proof thereof can be found in [Lu, $\S 1]$ ) that iteration of Rezk's construction yields, for every integer $n>1$, a left Bousfield localization of the Reedy structure on the category s ${ }^{n} \boldsymbol{S}$ of small $n$-simplicial spaces (i.e., $(n+1)$-simplicial sets) which is a model for the homotopy theory of $n$-fold homotopy theories, i.e., homotopy theories of ... of homotopy theories.

We will call the weak equivalences in these left Bousfield localization (which are often referred to as complete Segal equivalences) just Rezk equivalences.

Rezk's original result also gave rise to the following result on

\subsection{Relative categories}

Recall that a relative category is a pair $(\boldsymbol{C}, w \boldsymbol{C})$ consisting of a category $\boldsymbol{C}$ and a subcategory $w \boldsymbol{C} \subset \boldsymbol{C}$ which contains all the objects of $\boldsymbol{C}$ and of which the maps are called weak equivalences.

Received November 20, 2011, revised February 23, 2013; published on December 1, 2013. 2010 Mathematics Subject Classification: 18G55, 18D05.

Key words and phrases: relative category, $n$-relative category, higher category.

Article available at http://intlpress.com/HHA/v15/n2/a17 and doi:10.4310/HHA.2013.v15.n2.a17

Copyright (C) 2013, International Press. Permission to copy for private use granted. 
Then it was shown in [BK1] that Rezk's model structure on $\mathrm{s} \boldsymbol{S}$ (1.1) can be lifted to a Quillen equivalent Rezk structure on the category RelCat of the small relative categories, the weak equivalences of which will also (1.1) be called Rezk equivalences.

The category RelCat is connected to s $\boldsymbol{S}$ by a simplicial nerve functor

$$
N: \text { RelCat } \rightarrow \mathrm{s} \boldsymbol{S}
$$

with the property that a map $f \in \mathbf{R e l C a t}$ is a Rezk equivalence iff the map $N f \in \mathrm{s} \boldsymbol{S}$ is so. Moreover if we denote by $\mathbf{R k}$ the subcategories of the Rezk equivalences in both RelCat and $\mathrm{s} \boldsymbol{S}$, then the simplicial nerve functor has the property that

(i) the relative functor

$$
N:(\mathbf{R e l C a t}, \mathbf{R k}) \longrightarrow(\mathrm{s} \boldsymbol{S}, \mathbf{R k})
$$

is a homotopy equivalence of relative categories, in the sense that there exists a relative functor

$$
M:(\mathrm{s} \boldsymbol{S}, \mathbf{R k}) \longrightarrow(\mathbf{R e l C a t}, \mathbf{R k})
$$

called a homotopy inverse of $N$ such that the compositions $M N$ and $N M$ can be connected to the identity functors of RelCat and $s \boldsymbol{S}$ by finite zigzags of natural weak equivalences.

This in turn implies that

(ii) the relative category (RelCat, $\mathbf{R k})$ is, just like ( $\mathbf{S}, \mathbf{R k})$, a model for the homotopy theory of homotopy theories.

The proof of all this is essentially a relative version of the proof of the following classical result of Bob Thomason.

\subsection{Thomason's result}

In [T] Bob Thomason lifted the usual model structure on the category $\boldsymbol{S}$ of small spaces (i.e., simplicial sets) to a Quillen equivalent one on the category Cat of small categories and noted that these two categories were connected by the nerve functor $N$ : Cat $\rightarrow \boldsymbol{S}$ which has the property that a map $f \in \mathbf{C a t}$ is a weak equivalence iff $N f \in \boldsymbol{S}$ is so. It follows that, if $\boldsymbol{W}$ denotes the categories of weak equivalences in both $\mathbf{C a t}$ and $\boldsymbol{S}$, then

(i) the relative functor $N$ : (Cat, $\boldsymbol{W}) \rightarrow(\boldsymbol{S}, \boldsymbol{W})$ is a homotopy equivalence of relative categories (1.2(i))

which in turn implies that

(ii) the relative category $(\mathbf{C a t}, \boldsymbol{W})$ is, just like $(\boldsymbol{S}, \boldsymbol{W})$ a model for the theory of homotopy types.

His proof was however far from simple as it involved notions like two-fold subdivision and so-called Dwyer maps.

We end by recalling 


\subsection{A result of Dana Latch}

In [La] Dana Latch noted that, if one just wanted to prove 1.3(i) and 1.3(ii), one could do this by an argument that was much simpler than Thomason's and that, instead of the cumbersome two-fold subdivisions and Dwyer maps, involved the rather natural notion of the category of simplices of a simplicial set.

Now we can finally discuss

\subsection{The current paper}

The results mentioned in 1.1 and 1.2 above suggest that, for every integer $n>1$, there might exist some generalization of the notion of a relative category such that the category of such generalized relative categories admits a model structure which is Quillen equivalent to the Rezk structure on $\mathrm{s}^{n} \boldsymbol{S}$.

As however we did not see how to attack this question we turned to a much simpler one suggested by the result of Dana Latch that was mentioned in 1.4 above, namely to prove 1.2(i) directly by showing that

- the simplicial nerve functor

$$
N:(\mathbf{R e l C a t}, \mathbf{R k}) \longrightarrow(\mathrm{s} \boldsymbol{S}, \mathbf{R k})
$$

has an appropriately defined relative category of bisimplices functor

$$
K_{\delta}:(\mathrm{s} \boldsymbol{S}, \mathbf{R k}) \longrightarrow(\text { RelCat }, \mathbf{R k})
$$

as a homotopy inverse.

It turned out that not only could we do this, but the relative simplicity of our proof suggested that a similar proof might work for appropriately generalized relative categories. And indeed, after the necessary trial and error and frustration, we discovered a notion of what we will call $n$-relative categories which fitted the bill.

Hence the current manuscript.

\subsection{An overview}

There are six more sections.

In section 2 we introduce the category $\operatorname{Rel}^{n} \mathbf{C a t}$ of $n$-relative categories and in section 3 we formulate our main result. This involves

- The $n$-simplicial nerve functor $N: \operatorname{Rel}^{n} \mathbf{C a t} \rightarrow \mathrm{s}^{n} \boldsymbol{S}$ (1.1), and

- the $n$-relative category of multisimplices functor $K_{\delta}: \mathrm{s}^{n} \boldsymbol{S} \rightarrow \boldsymbol{R e l}^{n} \mathbf{C a t}$

and states that

- these two functors are homotopy inverses (1.2) of each other with respect to the Rezk structure on $\mathrm{s}^{n} \boldsymbol{S}$ (1.1) and the induced (by $N$ ) Rezk structure on Rel $^{n}$ Cat

which implies that

- $\boldsymbol{R e l}^{n}$ Cat, just like $\mathrm{s}^{n} \boldsymbol{S}$ (1.1), is a model for the homotopy theory of $n$-fold homotopy theories.

In section 4 we then prove this result modulo two key lemmas which we prove in sections 5 and 6 respectively. 
- The first lemma states the property that we wanted $n$-relative categories to have, namely that the associated $n$-simplicial nerve functor, just like the classical nerve functor, has a left adjoint which is a left inverse, and which after the necessary trial and error led to the current definition.

- The second lemma is the key ingredient in the proof of our main result. It states that the composite functor $N K_{\delta}$ preserves colimits and is an $n$-relative version of the key ingredient in Dana Latch's result (1.4) [La, Lemma B].

Finally in an appendix (section 7) we describe two relations between the categories $\mathbf{R e l}^{n} \mathbf{C a t}$ and $\mathbf{R e l}^{n+1}$ Cat which one would expect higher homotopy theories to have.

\section{Acknowledgments}

Chris Schommer-Pries closely read an earlier version of this paper and offered many useful comments. His observations and insights have led to major changes in the structure of our argument, and our understanding of $n$-relative categories has grown significantly as a result. We are grateful to him for his help.

\section{2. $n$-Relative categories}

After a brief review of relative categories we

- introduce $n$-relative categories $(n \geqslant 0)$ and

- describe some simple but useful examples which we will need in the next section.

\subsection{Relative categories}

A relative category is a pair $(\boldsymbol{C}, \boldsymbol{W})$ (often denoted by just $\boldsymbol{C}$ ) consisting of a category $\boldsymbol{C}$ (the underlying category) and a subcategory $\boldsymbol{W} \subset \boldsymbol{C}$, the maps of which are called the weak equivalences, and which is only subject to the condition that it contains all the objects of $\boldsymbol{C}$ (and hence all the identity maps).

The category of small relative categories and the relative (i.e., weak equivalence preserving) functors between them will be denoted by RelCat.

Two relative functors $\boldsymbol{C} \rightarrow \boldsymbol{D}$ are called naturally weakly equivalent if they can be connected by a finite zigzag of natural weak equivalences and a relative functor $f: \boldsymbol{C} \rightarrow \boldsymbol{D}$ will be called a homotopy equivalence if there exists a relative functor $g: \boldsymbol{D} \rightarrow \boldsymbol{C}$ (called a homotopy inverse of $f$ ) such that the compositions $g f$ and $f g$ are naturally weakly equivalent to $1_{\boldsymbol{C}}$ and $1_{\boldsymbol{D}}$ respectively.

\subsection{What to look for in a generalization}

In trying to generalize the notion of a relative category we were looking for

* a notion of $n$-relative category for which the associated $n$-simplicial nerve functor to $n$-simplicial spaces, just like the classical nerve functor, has a left adjoint which is also a left inverse.

Motivated by the fact that in an $n$-simplicial space (i.e., an $(n+1)$-simplicial set), just like in a simplicial space, the "space direction" plays a different role than "the $n$ 
simplicial directions", we start with considering sequences

$$
\boldsymbol{C}=\left(a \boldsymbol{C}, v_{1} \boldsymbol{C}, \ldots, v_{n} \boldsymbol{C}, w \boldsymbol{C}\right)(n \geqslant 0)
$$

consisting of a category $a \boldsymbol{C}$ and subcategories $v_{1} \boldsymbol{C}, \ldots, v_{n} \boldsymbol{C}$ and $w \boldsymbol{C} \subset a \boldsymbol{C}$, each of which contains all the objects of $a \boldsymbol{C}$ and which together with $a \boldsymbol{C}$ form a commutative diagram with $2 n$ arrows of the form

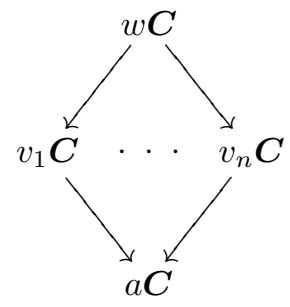

Such a sequence can be considered to consist of $n$ relative categories $v_{1} \boldsymbol{C}, \ldots, v_{n} \boldsymbol{C}$ which each has the same category of weak equivalences $w \boldsymbol{C}$ and an ambient category $a \boldsymbol{C}$ which encodes the relations between the $v_{i} \boldsymbol{C}(1 \leqslant i \leqslant n)$.

However the associated $n$-simplicial nerve functor (3.1) will only recognize those maps in $a \boldsymbol{C}$ which are finite compositions of maps in the $v_{i} \boldsymbol{C}(1 \leqslant i \leqslant n)$ and only those relations which are a consequence of the commutativity of those squares in $a \boldsymbol{C}$ which are of the form

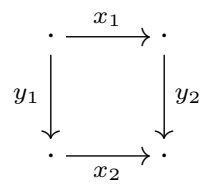

in which $x_{1}, x_{2} \in v_{i} \boldsymbol{C}$ and $y_{1}, y_{2} \in v_{j} \boldsymbol{C}$ (where $i$ and $j$ are not necessarily distinct).

In order that the associated $n$-simplicial nerve functor has a left adjoint which is a left inverse we therefore have to impose some restrictions on $a \boldsymbol{C}$ and define $n$-relative categories as follows.

\section{3. $\quad n$-Relative categories}

An $n$-relative category $\boldsymbol{C}(n \geqslant 0)$ will be an $(n+2)$-tuple

$$
\boldsymbol{C}=\left(a \boldsymbol{C}, v_{1} \boldsymbol{C}, \ldots, v_{n} \boldsymbol{C}, w \boldsymbol{C}\right)
$$

consisting of a category $a \boldsymbol{C}$ and subcategories

$$
v_{1} \boldsymbol{C}, \ldots, v_{n} \boldsymbol{C} \text { and } w \boldsymbol{C} \subset a \boldsymbol{C}
$$

each of which contains all the objects of $\boldsymbol{C}$ and which form a commutative diagram with $2 n$ arrows of the form

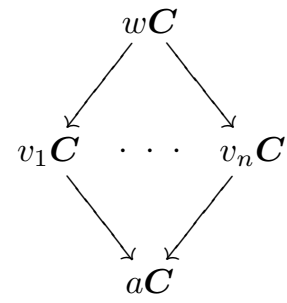


and where $a \boldsymbol{C}$ is subject to the condition that

(i) every map in $a \boldsymbol{C}$ is a finite composition of maps in the $v_{i} \boldsymbol{C}(1 \leqslant i \leqslant n)$, and

(ii) every relation in $a \boldsymbol{C}$ is a consequence of the commutativity relations in the $v_{i} C$ $(1 \leqslant i \leqslant n)$ and the commutativity of those squares in $a \boldsymbol{C}$ which are of the form

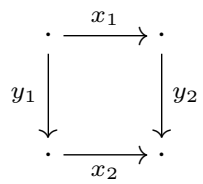

in which $x_{1}, x_{2} \in v_{i} \boldsymbol{C}$ and $y_{1}, y_{2} \in v_{j} \boldsymbol{C}$ and $1 \leqslant i<j \leqslant n$.

In particular

(iii) if $z_{i} \in v_{i} \boldsymbol{C}$ and $z_{j} \in v_{j} \boldsymbol{C}(i \neq j)$ are such that $z_{i}=z_{j}$, then this is a consequence of the commutativity of the square

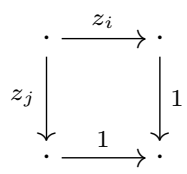

\subsection{Some comments}

In an $n$-relative category $\boldsymbol{C}$, the categories $v_{1} \boldsymbol{C}, \ldots, v_{n} \boldsymbol{C}$ are relative categories which have $w \boldsymbol{C}$ as their category of weak equivalences, and we will therefore sometimes refer to the maps of $w \boldsymbol{C}$ as weak equivalences.

Moreover the category $a \boldsymbol{C}$ is more than a common underlying category for the $v_{i} \boldsymbol{C}(1 \leqslant i \leqslant n)$ (as it may contain additional relations) and will therefore be called the ambient category.

Also note that

* A 1-relative category $\boldsymbol{C}$ is essentially just an ordinary relative category, as in that case a $\boldsymbol{C}=v_{1} \boldsymbol{C}$

and that

* a 0-relative category $\boldsymbol{C}$ is essentially a maxiamal relative category, i.e., a relative category in which all maps are weak equivalences, as in that case $a \boldsymbol{C}=w \boldsymbol{C}$.

\subsection{Relative functors}

A relative functor $f: \boldsymbol{C} \rightarrow \boldsymbol{D}$ between two $n$-relative categories $\boldsymbol{C}$ and $\boldsymbol{D}$ will be a functor $f: a \boldsymbol{C} \rightarrow a \boldsymbol{D}$ such that

$$
f w \boldsymbol{C} \subset w \boldsymbol{D} \quad \text { and } \quad f v_{i} \boldsymbol{C} \subset v_{i} \boldsymbol{D} \quad \text { for all } 1 \leqslant i \leqslant n .
$$

We will denote by $\mathbf{R e l}^{n} \mathbf{C a t}$ the resulting category of the small $n$-relative categories and the relative functors between them.

\subsection{Some examples}

Some rather simple but useful examples of $n$-relative categories are the following. 
For every integer $p \geqslant 0$ let $\boldsymbol{p}$ denote the category

$$
0 \longrightarrow \cdots \longrightarrow p
$$

and let $|\boldsymbol{p}| \subset \boldsymbol{p}$ be its subcategory which consists of the objects and their identity maps only. Then we will denote

(i) by $\boldsymbol{p}^{w} \in \boldsymbol{R e}^{n}$ Cat the object such that

$$
{ }_{a} \boldsymbol{p}^{w}=v_{i} \boldsymbol{p}^{w}=w \boldsymbol{p}^{w}=\boldsymbol{p} \quad \text { for all } 1 \leqslant i \leqslant n
$$

and

(ii) by $\boldsymbol{p}^{v_{i}} \in \operatorname{Rel}^{n}$ Cat the object such that

$$
a \boldsymbol{p}^{v_{i}}=v_{i} \boldsymbol{p}^{v_{i}}=\boldsymbol{p} \quad \text { and } \quad v_{j} \boldsymbol{p}^{v_{i}}=w \boldsymbol{p}^{v_{i}}=|\boldsymbol{p}| \quad \text { for } j \neq i .
$$

A simple calculation then yields that, for every sequence of integers $p_{n}, \ldots, p_{1}$, $q \geqslant 0$,

(iii) $\boldsymbol{p}_{n}^{v_{n}} \times \cdots \times \boldsymbol{p}_{1}^{v_{1}} \in \mathbf{R e l}^{n} \mathbf{C a t}$ is such that

$$
\begin{aligned}
a\left(\boldsymbol{p}_{n}^{v_{n}} \times \cdots \times \boldsymbol{p}_{1}^{v_{1}}\right) & =\boldsymbol{p}_{n} \times \cdots \times \boldsymbol{p}_{1} \\
w\left(\boldsymbol{p}_{n}^{v_{n}} \times \cdots \times \boldsymbol{p}_{1}^{v_{1}}\right) & =\left|\boldsymbol{p}_{n}\right| \times \cdots \times\left|\boldsymbol{p}_{1}\right| \quad \text { and } \\
v_{i}\left(\boldsymbol{p}_{n}^{v_{n}} \times \cdots \times \boldsymbol{p}_{1}^{v_{1}}\right) & =\left|\boldsymbol{p}_{n}\right| \times \cdots \times \boldsymbol{p}_{i} \times \cdots \times\left|\boldsymbol{p}_{1}\right| \quad(1 \leqslant i \leqslant n)
\end{aligned}
$$

and

(iv) $\boldsymbol{p}_{n}^{v_{n}} \times \cdots \times \boldsymbol{p}_{1}^{v_{1}} \times \boldsymbol{q}^{w} \in \mathbf{R e l}^{n} \mathbf{C a t}$ is such that

$$
\begin{aligned}
a\left(\boldsymbol{p}_{n}^{v_{n}} \times \cdots \times \boldsymbol{p}_{1}^{v_{1}} \times \boldsymbol{q}^{w}\right) & =\boldsymbol{p}_{n} \times \cdots \times \boldsymbol{p}_{1} \times \boldsymbol{q} \\
w\left(\boldsymbol{p}_{n}^{v_{n}} \times \cdots \times \boldsymbol{p}_{1}^{v_{1}} \times \boldsymbol{q}^{w}\right) & =\left|\boldsymbol{p}_{n}\right| \times \cdots \times\left|\boldsymbol{p}_{1}\right| \times \boldsymbol{q} \quad \text { and } \\
v_{i}\left(\boldsymbol{p}_{n}^{v_{n}} \times \cdots \times \boldsymbol{p}_{1}^{v_{1}} \times \boldsymbol{q}^{w}\right) & =\left|\boldsymbol{p}_{n}\right| \times \cdots \times \boldsymbol{p}_{i} \times \cdots \times\left|\boldsymbol{p}_{1}\right| \times \boldsymbol{q} \quad(1 \leqslant i \leqslant n)
\end{aligned}
$$

Moreover we will denote

(v) by $\delta \boldsymbol{p}^{v_{i}}(1 \leqslant i \leqslant n)$ and $\delta \boldsymbol{p}^{w} \in \mathbf{R e l}^{n}$ Cat the objects obtained by applying the following construction to the above $\boldsymbol{p}_{i}^{v_{i}}(1 \leqslant i \leqslant n)$ and $\boldsymbol{p}^{w}$.

\subsection{The (terminal) division of an $n$-relative category}

Motivated by the (terminal) subdivision of a relative category [BK1, 4.2], we define, for an object $\boldsymbol{C} \in \mathbf{R e l}^{n} \mathbf{C a t}$, its division $\delta \boldsymbol{C} \in \mathbf{R e l}^{n} \mathbf{C a t}$ as follows.

(i) $a \delta \boldsymbol{C}$ is the category which as as objects the functors $\boldsymbol{p} \rightarrow a \boldsymbol{C}(p \geqslant 0)$ and as maps

$$
\left(x_{1}: \boldsymbol{p}_{1} \rightarrow a \boldsymbol{C}\right) \longrightarrow\left(x_{2}: \boldsymbol{p}_{2} \rightarrow a \boldsymbol{C}\right)
$$

the commutative diagrams of the form

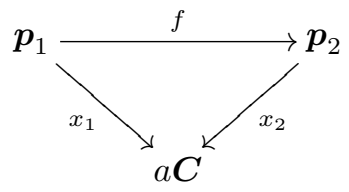

and 
(ii) $v_{i} \delta \boldsymbol{C}(1 \leqslant i \leqslant n)$ and $w \delta \boldsymbol{C}$ consists of those maps as in (i) for which the induced map

$$
x_{1} p_{1}=x_{2} f p_{1} \longrightarrow x_{2} p_{2}
$$

is in $v_{i} \boldsymbol{C}$ or $w \boldsymbol{C}$ respectively.

Clearly $\delta \boldsymbol{C}$ is natural in $\boldsymbol{C}$.

Moreover

(iii) $\delta \boldsymbol{C}$ comes with a natural (terminal) projection map

$$
\pi_{t}: \delta \boldsymbol{C} \longrightarrow \boldsymbol{C} \in \mathbf{R e l}^{n} \mathbf{C a t}
$$

which sends each object $x: \boldsymbol{p} \rightarrow \boldsymbol{C} \in \delta \boldsymbol{C}$ to the object $x p \in \boldsymbol{C}$ and which clearly has the following property:

\subsection{An easy result}

$A$ map $f \in \delta \boldsymbol{C}$ is in $v_{i} \delta \boldsymbol{C}(1 \leqslant i \leqslant n)$ or $w \delta \boldsymbol{C}$ iff $\pi_{t} f$ is in $v_{i} \boldsymbol{C}$ or $w \boldsymbol{C}$ respectively.

\section{Formulation of the main result}

To formulate our main result we need the following three notions.

\subsection{The $n$-simplicial nerve functor}

The $n$-simplicial nerver functor will be the right adjoint in the adjunction (1.1 and 2.5)

$$
K: \mathrm{s}^{n} \boldsymbol{S} \longleftrightarrow \operatorname{Rel}^{n} \text { Cat }: N \quad(n \geqslant 0)
$$

in which

(i) $N$ sends an object $\boldsymbol{C} \in \mathbf{R e l}^{n} \mathbf{C a t}$ to the $(n+1)$-simplicial set which has as its $\left(p_{n}, \ldots, p_{1}, q\right)$-simplices $\left(p_{n}, \ldots, p_{1}, q \geqslant 0\right)$ the maps $(2.6)$

$$
\boldsymbol{p}_{n}^{v_{n}} \times \cdots \times \boldsymbol{p}_{1}^{v_{1}} \times \boldsymbol{q}^{w} \longrightarrow \boldsymbol{C} \in \operatorname{Rel}^{n} \mathbf{C a t}
$$

and

(ii) $K$ is the colimit preserving functor which, for every $n+1$ integers $p_{n}, \ldots, p_{1}, q \geqslant$ 0 , sends the standard $\left(p_{n}, \ldots, p_{1}, q\right)$-simplex $\Delta\left[p_{n}, \ldots, p_{1}, q\right]$ to

$$
\boldsymbol{p}_{n}^{v_{n}} \times \cdots \times \boldsymbol{p}_{1}^{v_{n}} \times \boldsymbol{q}^{w} \in \mathbf{R e l}^{n} \mathbf{C a t}
$$

\subsection{The $n$-relative categories of multisimplicies functor}

The $n$-relative categories of multisimplices functor will be the colimit preserving functor

$$
K_{\delta}: \mathrm{s}^{n} \boldsymbol{S} \longrightarrow \operatorname{Rel}^{n} \mathbf{C a t} \quad(n \geqslant 0)
$$

which sends each standard multisimplex $\Delta\left[p_{n}, \ldots, p_{1}, q\right]\left(p_{n}, \ldots, p_{1}, q \geqslant 0\right)$ to the object (2.6)

$$
\delta \boldsymbol{p}_{n}^{v_{n}} \times \cdots \times \delta \boldsymbol{p}_{1}^{v_{1}} \times \delta \boldsymbol{q}^{w} \in \mathbf{R e l}^{n} \mathbf{C a t},
$$

It comes with a (terminal) projection map which, with a slight abuse of notation, we denote by

$$
\pi_{t}: K_{\delta} \longrightarrow K
$$


and which is induced by the $(n+1)$-fold (terminal) projection map 2.7(iii)

$$
\delta \boldsymbol{p}_{n}^{v_{n}} \times \cdots \delta \boldsymbol{p}_{1}^{v_{1}} \times \delta \boldsymbol{q}^{w} \stackrel{\pi_{t} \times \cdots \times \pi_{t}}{\longrightarrow} \boldsymbol{p}_{n}^{v_{n}} \times \cdots \boldsymbol{p}_{1}^{v_{1}} \times \boldsymbol{q}^{w}
$$

\subsection{Reedy and Rezk equivalences in $\operatorname{Rel}^{n}$ Cat}

A map $f \in \operatorname{Rel}^{n}$ Cat $(n \geqslant 1)$ will be called a Reedy or a Rezk equivalence iff the map $n f \in \mathrm{s}^{n} \boldsymbol{S}$ is (1.1), and we will denote by

$$
\mathbf{R y} \text { and } \mathbf{R k} \subset \mathrm{s}^{n} \boldsymbol{S} \quad \text { and } \quad \mathbf{R y} \text { and } \mathbf{R k} \subset \mathbf{R e l}^{n} \mathbf{C a t}
$$

the subcategories of the Reedy and Rezk equivalences.

We can now state our main result.

\subsection{Main Theorem}

(i) The functors $N$ and $K_{\delta}$ induce inverse homotopy equivalences (2.1)

$$
N:\left(\mathbf{R e l}^{n} \mathbf{C a t}, \mathbf{R y}\right) \leftrightarrows\left(\mathrm{s}^{n} \boldsymbol{S}, \mathbf{R y}\right): K_{\delta}
$$

which, in view of the fact that the Rezk equivalences in $\mathrm{s}^{n} \boldsymbol{S}$ are the weak equivalences in a left Bousfield localization of the Reedy structure, implies that

(ii) they also induce homotopy equivalences

$$
N:\left(\boldsymbol{R e l}^{n} \mathbf{C a t}, \mathbf{R k}\right) \leftrightarrows\left(\mathrm{s}^{n} \boldsymbol{S}, \mathbf{R k}\right): K_{\delta}
$$

which means that

(iii) just like $\left(\mathrm{s}^{n} \boldsymbol{S}, \mathbf{R k}\right)(1.1)$, (Rel $\left.{ }^{n} \mathbf{C a t}, \mathbf{R k}\right)$ is a homotopy theory of $n$-fold homotopy theories.

\section{A proof of our main result}

We start with a proof of several auxiliary propositions and the formulation of two key lemmas which we will prove in sections 5 and 6 respectively.

\subsection{Proposition}

For every standard multisimplex $\Delta\left[p_{n}, \ldots, p_{1}, q\right] \in \mathrm{s}^{n} \boldsymbol{S}(n \geqslant 1)$, the map (3.2)

$$
N \pi_{t}: N K_{\delta} \Delta\left[p_{n}, \ldots, p_{1}, q\right] \longrightarrow N K \Delta\left[p_{n}, \ldots, p_{1}, q\right] \in \mathrm{s}^{n} \boldsymbol{S}
$$

is a Reedy equivalence.

Proof. As

$$
K_{\delta} \Delta\left[p_{n}, \ldots, p_{1}, q\right]=\delta \boldsymbol{p}_{n}^{v_{n}} \times \cdots \times \delta \boldsymbol{p}_{1}^{v_{1}} \times \delta \boldsymbol{q}^{w}
$$

and

$$
K \Delta\left[p_{n}, \ldots, p_{1}, q\right]=\boldsymbol{p}_{n}^{v_{n}} \times \cdots \times \boldsymbol{p}_{1}^{v_{1}} \times \boldsymbol{q}^{w}
$$

we have to prove that application of the functor $N$ to the map in $3.2(*)$ above yields a Reedy equivalence. But $N$ is a right adjoint and hence preserves products. Since 
Reedy equivalences are stable under products, it suffices to show that each of the maps

$$
N \pi_{t}: N \delta \boldsymbol{p}_{i}^{v_{i}} \longrightarrow N \boldsymbol{p}_{i}^{v_{i}} \quad \text { and } \quad N \pi_{t}: N \delta \boldsymbol{a}^{w} \longrightarrow N \boldsymbol{q}^{w}
$$

is a Reedy equivalence.

To do this let

$$
\tau: \boldsymbol{p}^{v_{i}} \longrightarrow \delta \boldsymbol{p}^{v_{i}} \quad \text { and } \quad \tau: \boldsymbol{p}^{w} \longrightarrow \delta \boldsymbol{p}^{w}
$$

be the maps which send an object $b \in \boldsymbol{p}$ to the object

$$
\boldsymbol{b}=(0 \rightarrow \cdots \rightarrow b) \stackrel{\text { incl. }}{\longrightarrow}(0 \rightarrow \cdots \rightarrow p) \in \delta \boldsymbol{p}^{v_{i}} \text { or } \delta \boldsymbol{p}^{w} .
$$

Then $\pi_{t} \tau=1$ and there are obvious maps

$$
h: \delta \boldsymbol{p}^{v_{i}} \times \mathbf{1}^{w} \longrightarrow \delta \boldsymbol{p}_{v_{i}} \quad \text { and } \quad h: \delta \boldsymbol{p}^{w} \times \mathbf{1}^{w} \longrightarrow \delta \boldsymbol{p}^{w}
$$

such that $h 0=1$ and $h 1=\tau \pi_{t}$.

The desired result then follows readily from the observation that if

(i) two maps $f, g: \boldsymbol{C} \rightarrow \boldsymbol{D} \in \mathbf{R e l}^{n}$ Cat are strictly homotopic in the sense that there exists a map $h: \boldsymbol{C} \times \mathbf{1}^{w} \rightarrow \boldsymbol{D} \in \mathbf{R e l}^{n} \mathbf{C a t}$ connecting them,

then

(ii) the maps $N f, N g: N \boldsymbol{C} \rightarrow N \boldsymbol{D} \in \mathrm{s}^{n} \boldsymbol{S}$ are strictly homotopic in the sense that there exists a map $k: N \boldsymbol{C} \times \Delta[0, \ldots, 0,1] \rightarrow N \boldsymbol{D} \in \mathrm{s}^{n} \boldsymbol{S}$ connecting them, where

(iii) $k$ is the composition

$$
\begin{aligned}
N \boldsymbol{C} \times \Delta[0, \ldots, 1] \stackrel{\eta}{\longrightarrow} N \boldsymbol{C} \times N K \Delta[0, \ldots, 0,1] \\
\stackrel{\mathrm{Id}}{\longrightarrow} N \boldsymbol{C} \times N \mathbf{1}^{w} \stackrel{\approx}{\longrightarrow} N\left(\boldsymbol{C} \times \mathbf{1}^{w}\right) \stackrel{h}{\longrightarrow} N \boldsymbol{D}
\end{aligned}
$$

\subsection{Proposition}

For every standard multisimplex $\Delta\left[p_{n}, \ldots, p_{1}, q\right] \in \mathrm{s}^{n} \boldsymbol{S}$ the unit map (3.1)

$$
\eta \Delta\left[p_{n}, \ldots, p_{1}, q\right]: \Delta\left[p_{n}, \ldots, p_{1}, q\right] \longrightarrow N K \Delta\left[p_{n}, \ldots, p_{1}, q\right] \in \mathrm{s}^{n} \boldsymbol{S}
$$

is a Reedy equivalence.

Proof. Note that

$$
\Delta\left[p_{n}, \ldots, p_{1}, q\right]=\Delta\left[p_{n},-\right] \times \cdots \times \Delta\left[-, p_{i},-\right] \times \cdots \times \Delta[-, q]
$$

where - denotes a (possibly empty) sequence of 0's. A straightforward calculation then yields that the map

$$
\eta \Delta\left[-, p_{i},-\right]: \Delta\left[-, p_{i},-\right] \longrightarrow N K \Delta\left[-, p_{i},-\right]=N p_{i}^{v_{i}} \in \mathrm{s}^{n} \boldsymbol{S}
$$

is an isomorphism for all $1 \leqslant i \leqslant n$ and that

$$
\eta \Delta][-, q]: \Delta[-, q] \longrightarrow N K \Delta[-, q] \in \mathrm{s}^{n} \boldsymbol{S}
$$

is a Reedy equivalence and the desired result now follows from the fact that $N$ is a right adjoint and hence preserves products and therefore Reedy equivalences. 


\subsection{The first key lemma}

For every object $\boldsymbol{C} \in \boldsymbol{R}^{n} \mathbf{l}^{n} \mathbf{C a t}(n \geqslant 0$ ) the counit map (3.1)

$$
\varepsilon \boldsymbol{C}: K N \boldsymbol{C} \longrightarrow \boldsymbol{C} \in \mathbf{R e l}^{n} \mathbf{C a t}
$$

is an isomorphism, i.e., $K$ is not only a left adjoint but also a left inverse of $N$.

A proof will be given in section 5 .

We call this a key lemma because it states the property that we wanted $n$-relative categories to have and which therefore led to the, a priori mysterious, restriction imposed in 2.3(ii).

Our other preliminary results involve the following notion.

\subsection{The (ordinary) categories of multisimplices functor}

Let $\Delta[-] \subset \mathrm{s}^{n} \boldsymbol{S}(n \geqslant 0)$ denote the full cubcategory spanned by the standard multisimplices.

Given an object $X \in \mathrm{s}^{n} \boldsymbol{S}$, its (ordinary) category of multisimplices $\Delta X$ then is defined as the overcategory

$$
\Delta X=\Delta[-] \downarrow X
$$

Clearly $\Delta X$ is natural in $X$ and comes with a forgetful functor

$$
F: \Delta X \longrightarrow \mathrm{s}^{n} \boldsymbol{S}
$$

which sends an object $\Delta\left[p_{n}, \ldots, p_{1}, q\right] \rightarrow X \in \Delta X$ to the object $\Delta\left[p_{n}, \cdots, p_{1}, q\right] \in$ $\mathrm{s}^{n} \boldsymbol{S}$.

\subsection{Proposition}

For every object $X \in \mathrm{s}^{n} \boldsymbol{S}$ the category $\Delta X$ is a Reedy category with fibrant constants $[\mathrm{H}, 15.10 .1(2)]$.

Proof. This is a straightforward calculation.

\subsection{Proposition}

For every object $X \in \mathrm{s}^{n} \boldsymbol{S}$, the obvious map

$$
\operatorname{colim}_{\Delta X} F \longrightarrow X \in \mathrm{s}^{n} \boldsymbol{S}
$$

is an isomorphism.

Proof. We have to show that in every multidimension $\left(s_{n}, \ldots, s_{1}, t\right)$ this map is 1-1 and onto.

That it is onto follows from the fact that, for every $\left(s_{n}, \ldots, s_{1}, t\right)$-simplex $e \in X$, the associated map $\bar{e}: \Delta\left[s_{n}, \ldots, s_{1}, t\right] \rightarrow X \in \mathrm{s}^{n} \boldsymbol{S}$ sends the generating multisimplex of the standard multisimplex to $e$.

That it is also 1-1 follows from the observation that every pair consisting of

(i) a map $\bar{f}: \Delta\left[p_{n}, \ldots, p_{1}, q\right] \rightarrow X \in \mathrm{s}^{n} \boldsymbol{S}\left(p_{n}, \ldots, p_{1}, q \geqslant 0\right)$ and

(ii) an $\left(s_{n}, \ldots, s_{1}, t\right)$-simplex $h \in \Delta\left[p_{n}, \ldots, p_{1}, q\right]$ such that $\bar{f} h=e$ 
gives rise to a commutative diagram

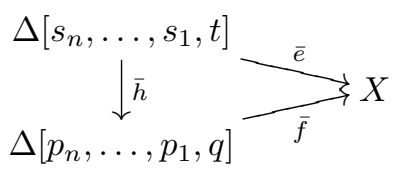

\subsection{The second key lemma}

For every object $X \in \mathrm{s}^{n} \boldsymbol{S}$ the obvious map

$$
\operatorname{colim}_{\Delta X} N K_{\delta} F \longrightarrow N K_{\delta} X \in \mathrm{s}^{n} \boldsymbol{S}
$$

is an isomorphism.

A proof will be given in section 6 .

We call this a key lemma because it is the key ingredient in the proof of our main result which is not surprising as it is an $n$-relative version of the lemma [La, Lemma B] of Dana Latch, which was the key ingredient in the proof of her result.

Now we are ready for a proof of our main result.

\subsection{A proof of theorem 3.4}

To prove that the functors $N K_{\delta}$ and $1_{\mathrm{s}^{n}} \boldsymbol{S}$ are naturally Reedy equivalent we consider, for every object $X \in \mathrm{s}^{n} \boldsymbol{S}$, the commutative diagram (3.1, 3.2, 4.2 and 4.4)

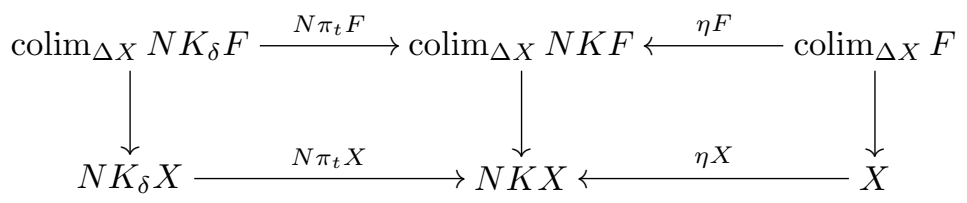

in which the vertical maps are the obvious ones.

The vertical maps on the outside are, in view of 4.7 and 4.6, isomorphisms and it thus suffices to prove that the upper maps are Reedy equivalences. But this follows immediately from 4.1, 4.2, 4.5 and the result [H, 15.10.9(2)] that the colimit of an objectwise weak equivalence between Reedy cofibrant diagrams indexed by a Reedy category with fibrant constants is also a weak equivalence.

Note that the fact that these four maps are Reedy equivalences also implies that the functor $N K_{\delta}$ preserves Reedy equivalences and so does therefore (3.3) the functor $K_{\delta}$.

To prove that the functors $K_{\delta} N$ and $1_{\mathbf{R e}^{n}} \mathbf{C a t}$ are also naturally Reedy equivalent it suffices to show that, for every object $\boldsymbol{C} \in \mathbf{R e l}^{n} \mathbf{C a t}$, both maps in the sequence

$$
K_{\delta} N \boldsymbol{C} \stackrel{\pi_{t} N \boldsymbol{C}}{\longrightarrow} K N C \stackrel{\varepsilon \boldsymbol{C}}{\longrightarrow} \boldsymbol{C} \in \operatorname{Rel}^{n} \mathbf{C a t}
$$

are Reedy equivalences. For the second map this follows from 4.3. To deal with the first one we have to show that $N \pi_{t} N \boldsymbol{C}$ is a Reedy equivalence in ${ }^{n} \boldsymbol{S}$. This we do by 
considering the above diagram for $X=N C$

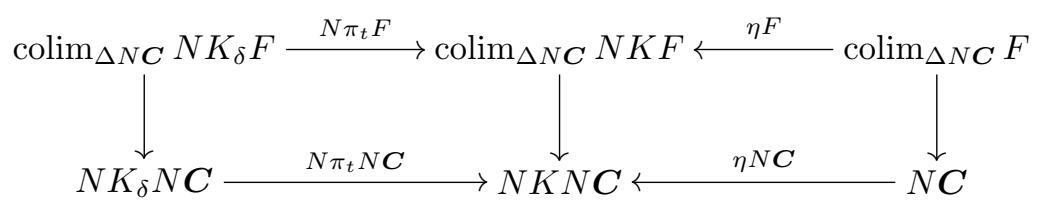

and then noting that all its maps are Reedy equivalences in view of the fact that

(i) the upper and the outside vertical maps are so by the above,

(ii) the map $\eta N \boldsymbol{C}$ is an isomorphism because $N \varepsilon \boldsymbol{C}$ (4.3) and the composition $N C \stackrel{\eta N C}{\longrightarrow} N K N C \stackrel{N \varepsilon C}{\longrightarrow} N C$ are so, and

(iii) Reedy equivalences have the two out of three property.

\section{A proof of the first key lemma (4.3)}

To prove our first key lemma we need an $n$-relative version of proposition 4.6 which involves the following notion of (ordinary) categories of multisimplices (of the $n$-simplicial nerve) of an $n$-relative category.

\subsection{The category of multisimplices of an $n$-relative category}

Given an object $\boldsymbol{C} \in \mathbf{R e l}^{n} \mathbf{C a t}$, its (ordinary) category of multisimplices $\Delta C$ is defined as the overcategory (3.1 and 4.4)

$$
\Delta C=K \Delta[-] \downarrow C
$$

Clearly $\Delta \boldsymbol{C}$ is natural in $\boldsymbol{C}$ and comes with a forgetful functor

$$
G: \Delta C \longrightarrow \operatorname{Rel}^{n} \mathbf{C a t}
$$

which sends an object $K \Delta\left[p_{n}, \ldots, p_{1}, q\right] \rightarrow \boldsymbol{C} \in \Delta \boldsymbol{C}$ to the object $K \Delta\left[p_{n}, \ldots, p_{1}, q\right] \in$ $\operatorname{Rel}^{n}$ Cat.

As a first step in proving the first key lemma we then note the following

\subsection{Proposition}

For every object $\boldsymbol{C} \in \mathbf{R e}^{n} \mathbf{C a t}$, the obvious map

$$
\operatorname{colim}_{\Delta \boldsymbol{C}} G \longrightarrow \boldsymbol{C} \in \mathbf{R e l}^{n} \mathbf{C a t}
$$

is an isomorphism.

Proof. We first note that

$$
a K \Delta\left[p_{n}, \ldots, p_{1}, q\right]=a\left(\boldsymbol{p}_{n}^{v_{n}} \times \cdots \times \boldsymbol{p}_{1}^{v_{1}} \times \boldsymbol{q}^{w}\right)=\boldsymbol{p}_{n} \times \cdots \times \boldsymbol{p}_{1} \times \boldsymbol{q}
$$

is a poset which has an object, a generating map and a relation for every subobject

$$
\boldsymbol{s}_{n} \times \cdots \times \boldsymbol{s}_{1} \times t \subset \boldsymbol{p}_{n} \times \cdots \times \boldsymbol{p}_{1} \times \boldsymbol{q}
$$

for which $s_{n}+\cdots+s_{1}+t$ is 0,1 or 2 .

This readily implies that 
* to compute $\operatorname{colim}_{\triangle C} G$ it suffices to restrict the computation to the full subcategory of $\Delta \boldsymbol{C}$ spanned by the maps into $\boldsymbol{C}$ from respectively the objects which are isomorphic to $(2.6)$

(i) $\mathbf{0}^{w}, \mathbf{1}^{w}$ and $\mathbf{1}^{v_{i}}(1 \leqslant i \leqslant n)$

(ii) $\mathbf{2}^{w}$ and $\mathbf{2}^{v_{i}}$ and $\mathbf{1}^{v_{i}} \times \mathbf{1}^{w}(1 \leqslant i \leqslant n)$, and

(iii) $\mathbf{1}^{v_{i}} \times \mathbf{1}^{v_{j}}(1 \leqslant i<j \leqslant n)$.

Arguments similar to the ones used in the proof of proposition 4.6 then yield that the maps mentioned in (i) above give rise to isomorphisms

$$
w \operatorname{colim}_{\Delta \boldsymbol{C}} G \approx w \boldsymbol{C} \text { and } v_{i} \operatorname{colim}_{\Delta \boldsymbol{C}} G \approx v_{i} \boldsymbol{C} \quad(1 \leqslant i \leqslant n) .
$$

As these isomorphisms are isomorphisms of categories it follow that the map mentioned in (ii) above do not give rise to any additional structure.

That these isomorphisms extend to isomorphisms colim ${ }^{\Delta C} G \approx C \in \operatorname{Rel}^{n} \mathbf{C a t}$ now follows from the fact that the maps mentioned in (iii) above give rise to exactly the commutative squares that were required by 2.3(ii) above.

The first key lemma can now be obtained by the following rather formal argument

\subsection{A proof of the first key lemma (4.3)}

Let $\Delta N \boldsymbol{C}$ and $F: \Delta N \boldsymbol{C} \rightarrow \mathrm{s}^{n} \boldsymbol{S}$ be as in 4.4. Then the adjunction $K \leftrightarrow N$ (3.1) gives rise to an isomorphism $\Delta N C \approx \Delta C$ which sends each map

$$
y: \Delta\left[p_{n}, \ldots, p_{1}, q\right] \longrightarrow N \boldsymbol{C} \in \mathrm{s}^{n} \boldsymbol{S}
$$

to the map

$$
K \Delta\left[p_{n}, \ldots, p_{1}, q\right] \longrightarrow \boldsymbol{C} \in \operatorname{Rel}^{n} \mathbf{C a t}
$$

which is the composition

$$
K \Delta\left[p_{n}, \ldots, p_{1}, q\right] \stackrel{K y}{\longrightarrow} K N C \stackrel{\varepsilon}{\longrightarrow} C
$$

whiere $\varepsilon$ denotes the counit of the adjunction.

These sequences combine to form the commutative square

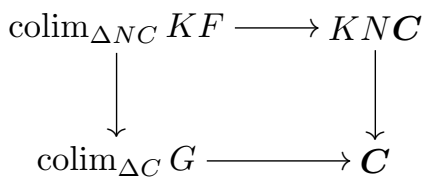

in which the map on the left is induced by the isomorphism between the diagrams $K F$ and $G$ and hence is an isomorphism. Moreover the map at the bottom was shown to be an isomorphism in 5.2 and this map of the top is an isomorphism in view of proposition 4.6 and the fact that the functor $K$ as a left adjoint commutes with colimits. Thus the map on the right is also an isomorphism.

\section{A proof of the second key lemma (4.7)}

We start with a result (6.1) which is a variation on proposition 4.6. 
Using this we then (in 6.2) give an alternate description of the $n$-relative categories of multisimplices functor (3.2) which brings out its close relationship with the (ordinary) categories of multisimplices functor of 4.4 .

This in turn we use (in 6.3) to prove what is in some sense a generalization of proposition 4.7 which we then use (in 6.4) to obtain proposition 4.7 itself.

\subsection{Proposition}

For every object $X \in \mathrm{s}^{n} \boldsymbol{S}$, the obvious map (4.6)

$$
\operatorname{colim}_{\Delta X} \Delta F \longrightarrow \Delta X \in \mathbf{C a t}
$$

is an isomorphism.

Proof. For every object $e: \Delta\left[s_{n}, \ldots, s_{1}, t\right] \rightarrow X \in \Delta X \quad\left(s_{n}, \ldots, s_{1}, t \geqslant 0\right) e$ is the image under the map $\Delta e: \Delta \Delta\left[s_{n}, \ldots, s_{1}, t\right] \rightarrow \Delta X$ of the identity map

$$
\Delta\left[s_{n}, \ldots, s_{1}, t\right] \rightarrow \Delta\left[s_{n}, \ldots, s_{1}, t\right] \in \Delta \Delta\left[s_{n}, \ldots, s_{1}, t\right] .
$$

This readily implies that the map $\operatorname{colim}_{\Delta X} \Delta F \rightarrow \Delta X$ is onto.

That it is also 1-1 follows from the observation that every pair consisting of

(i) a map $f: \Delta\left[p_{n}, \ldots, p_{1}, q\right] \rightarrow X \in \Delta X\left(p_{n}, \ldots, p_{1}, q \geqslant 0\right)$, and

(ii) an object $h: \Delta\left[s_{n}, \ldots, s_{1}, t\right] \rightarrow \Delta\left[p_{n}, \ldots, p_{1}, q\right] \in \Delta \Delta\left[p_{n}, \ldots, p_{1}, q\right]$ such that $f h=e$

gives rise to a commutative triangle

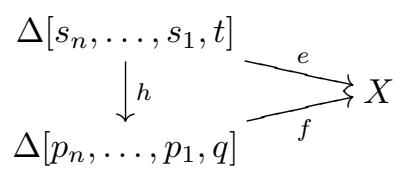

\subsection{An alternate description of $K_{\delta}$}

It follow readily from the definitions involved that

(i) for every sequence of cateogries $p_{n}, \ldots, p_{1}, q \geqslant 0$, there is a canonical isomorphism (4.4)

$$
a K_{\delta} \Delta\left[p_{n}, \ldots, p_{1}, q\right] \approx \Delta \Delta\left[p_{n}, \ldots, p_{1}, q\right]=\Delta[-] \downarrow \Delta\left[p_{n}, \ldots, p_{1}, q\right]
$$

Consequently one can

(ii) define an object $\Delta_{\text {rel }} \Delta\left[p_{n}, \ldots, p_{1}, q\right]$ that is canonically isomorphic to the object $K_{\delta} \Delta\left[p_{n}, \ldots, p_{1}, q\right]$ by endowing the object $\Delta \Delta\left[p_{n}, \ldots, p_{1}, q\right]$ with the $n$-relative structure lifted from $K_{\delta} \Delta\left[p_{n}, \ldots, p_{1}, q\right]$, and

(iii) define, for every object $X \in \mathrm{s}^{n} \boldsymbol{S}$, an object $\Delta_{\text {rel }} X \in \mathbf{R e l}^{n}$ Cat by the formula (4.4)

$$
\Delta_{\text {rel }} X=\operatorname{colim}_{\Delta X} \Delta_{\text {rel }} F
$$

As the functors $K_{\delta}$ and $\Delta_{\text {rel }}$ both preserve colimits it follows that

(iv) the functors $K_{\delta}$ and $\Delta_{\text {rel }}$ are canonically isomorphic.

Moreover it follows from proposition 6.1 above and the fact that the ambient category functor $a$ : $\mathbf{R e l}^{n} \mathbf{C a t} \rightarrow \mathbf{C a t}$ preserves colimits that 
(v) $a \Delta_{\text {rel }} X=\Delta X$ for all $X \in \mathrm{s}^{n} \boldsymbol{S}$.

Using this alternate definition we now can state the following

\subsection{A variation on proposition 4.7}

Assume that

(i) $\boldsymbol{T} \in \mathbf{R e l}^{n} \mathbf{C a t}$ and an object $T \in \boldsymbol{T}$ are such that $a \boldsymbol{T}$ is a poset and $T$ is a terminal object of $a \boldsymbol{T}$, and that

(ii) for every pair of objects $\boldsymbol{C}, \boldsymbol{D} \in \mathbf{R e l}^{n} \mathbf{C a t} \operatorname{map}(\boldsymbol{C}, \boldsymbol{D})$ denotes the set of maps $\boldsymbol{C} \rightarrow \boldsymbol{D} \in \operatorname{Rel}^{n}$ Cat.

Then

(iii) for every object $X \in \mathrm{s}^{n} \boldsymbol{S}$, the obvious map

$$
\operatorname{colim}_{\Delta X} \operatorname{map}\left(\boldsymbol{T}, \Delta_{\text {rel }} F\right) \longrightarrow \operatorname{map}\left(\boldsymbol{T}, \Delta_{\text {rel }} X\right)
$$

is an isomorphism.

Proof. That this map is onto follows readily from the fact that every map $e: \boldsymbol{T} \rightarrow$ $\Delta_{\text {rel }} X \in \mathbf{R e l}^{n} \mathbf{C a t}$ admits a unique factorization of the form

$$
\boldsymbol{T} \stackrel{e_{1}}{\longrightarrow} \Delta_{\mathrm{rel}} \Delta\left[s_{n}, \ldots, s_{1}, t\right] \stackrel{\Delta_{\mathrm{rel}} e_{2}}{\longrightarrow} \Delta_{\mathrm{rel}} X
$$

in which $e_{1}$ sends $T$ to the identity map of $\Delta\left[s_{n}, \ldots, s_{1}, t\right]$ and $e_{2}$ sends this map to $e T$.

That it is also 1-1 then follows from the observation that every pair consisting of

(i) a factorization of $e$ of the form

$$
\boldsymbol{T} \stackrel{f_{1}}{\longrightarrow} \Delta_{\mathrm{rel}} \Delta\left[p_{n}, \ldots, p_{1}, q\right] \stackrel{\Delta_{\mathrm{rel}} f_{2}}{\longrightarrow} \Delta_{\mathrm{rel}} X
$$

(ii) and an object

$$
h: \Delta\left[s_{n}, \ldots, s_{1}, t\right] \longrightarrow \Delta\left[p_{n}, \ldots, p_{1}, q\right] \in \Delta_{\text {rel }} \Delta\left[p_{n}, \ldots, p_{1}, q\right]
$$

such that $f_{2} h=e_{2}$

gives rise to a commutative diagram

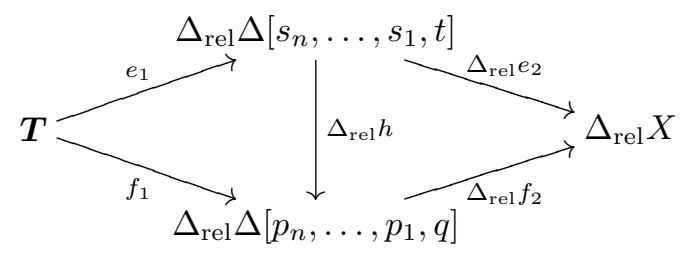

\subsection{A proof of the second key lemma (4.7)}

Proposition 4.7 now follows readily from 6.2 (iv) and proposition 6.3 above and the fact that the $n$-relative categories

$$
\boldsymbol{p}_{n}^{v_{n}} \times \cdots \times \boldsymbol{p}_{1}^{v_{1}} \times \boldsymbol{q}^{w}
$$

involved in the definition of the $n$-simplicial nerve functor $N(3.1)$ have the properties mentioned in 6.3(i) above. 


\section{Appendix}

In this appendix we note that the categories $\operatorname{Rel}^{n} \mathbf{C a t}(n \geqslant 1)$ have two additional properties which one would expect a homotopy theory of homotopy theories to have:

A. There there exists a functor $\operatorname{Rel}^{n} \mathbf{C a t} \rightarrow \mathbf{R e l}^{n+1} \mathbf{C a t}$ which has a left inverse right adjoint.

B. That every object of $\mathbf{R e l}^{n+1} \mathbf{C a t}$ gives rise to a category enriched over $\mathbf{R e l}^{n} \mathbf{C a t}$ which suggests the possibility that "a map in $\mathbf{R e l}^{n+1} \mathbf{C a t}$ is a Rezk equivalence (3.3) iff the induced map between these enriched categories is a kind of $D K$ equivalence".

To deal with A we note that a straightforward calculation yields:

\subsection{Proposition}

For every integer $n \geqslant 1$ the functor

$$
\operatorname{Rel}^{n} \mathbf{C a t} \longrightarrow \operatorname{Rel}^{n+1} \mathbf{C a t}
$$

which sends

$$
\left(a \boldsymbol{C}, v_{1} \boldsymbol{C}, \ldots, v_{n} \boldsymbol{C}, w \boldsymbol{C}\right) \quad \text { to } \quad\left(a \boldsymbol{C}, v_{1} \boldsymbol{C}, \ldots, v_{n} \boldsymbol{C}, w \boldsymbol{C}, w \boldsymbol{C}\right)
$$

has a right adjoint left inverse which sends

$$
\left(a \boldsymbol{D}, v_{1} \boldsymbol{D}, \ldots, v_{n+1} \boldsymbol{D}, w \boldsymbol{D}\right) \quad \text { to } \quad\left(\bar{a} \boldsymbol{D}, v_{1} \boldsymbol{D}, \ldots, v_{n} \boldsymbol{D}, w \boldsymbol{D}\right)
$$

where $\bar{a} \boldsymbol{D} \subset$ a $\boldsymbol{D}$ denotes the subcategory which consists of the finite compositions of maps in the $v_{i} \boldsymbol{D}(1 \leqslant i \leqslant n)$.

We deal with B by means of an $n$-relative version of the Grothendieck enrichment of [DHKS, 3.4 and 3.5].

To do this we start with recalling

\subsection{Types of zigzags}

The type of a zigzag of maps in a category $C$ from an object $X$ to an object $Y$

$$
X \stackrel{f_{1}}{X} \cdot \frac{f_{m}}{\quad} Y \quad(m \geqslant 0)
$$

is the pair $T=\left(T_{+}, T_{-}\right)$of complementary subsets of the set of integers $\{1, \ldots, m\}$ such that $i \in T_{+}$whenever $f_{i}$ is a forward map and $i \in T_{-}$otherwise.

These types can be considered as the objects of a category of types $\boldsymbol{T}$ which has, for every two types $\left(T_{+}, T_{-}\right)$and $\left(T_{+}^{\prime}, T_{-}^{\prime}\right)$ of length $m$ and $m^{\prime}$ respectively, as maps $t:\left(T_{+}, T_{-}\right) \rightarrow\left(T_{+}^{\prime}, T_{-}^{\prime}\right)$ the weakly monotonic maps $t:\{1, \ldots, m\} \rightarrow\left\{1, \ldots, m^{\prime}\right\}$ such that

$$
t T_{+} \subset T_{+}^{\prime} \quad \text { and } \quad t T_{-} \subset T_{-}^{\prime}
$$

With these types one then associates 


\section{3. $\quad n$-Relative arrow categories}

Given an object $\boldsymbol{C} \in \mathbf{R e l}^{n+1} \mathbf{C a t}$ let, as in $7.1, \bar{a} \boldsymbol{C} \subset a \boldsymbol{C}$ denote the subcategory which consists of the finite compositions of maps of the $v_{i} C(1 \leqslant i \leqslant n)$.

For every pair of objects $X, Y \in C$ and type $T$ (7.2) we then denote by $\boldsymbol{C}^{T}(X, Y) \in$ $\operatorname{Rel}^{n}$ Cat the $n$-relative arrow category which has

(i) as objects the zigzags of type $T$ in $\boldsymbol{C}$ between $X$ and $Y$ in which the backward maps are in $\bar{a} \boldsymbol{C}$,

(ii) as maps in $v_{i} \boldsymbol{C}^{T}(X, Y)(1 \leqslant i \leqslant n)$ and $w \boldsymbol{C}^{T}(X, Y)$ between two such zigzags the commutative diagrams of the form

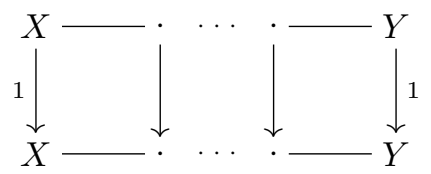

in which the vertical maps are in $v_{i} \boldsymbol{C}$ and $w \boldsymbol{C}$ respectively, and

(iii) as maps in $a \boldsymbol{C}^{T}(X, Y)$ the finite compositions of maps of the $v_{i} \boldsymbol{C}^{T}(X, Y)$ $(1 \leqslant i \leqslant n)$.

These arrow categories in turn give rise to

\section{4. $\quad T$-diagrams of arrow categories}

Given an object $\boldsymbol{C} \in \mathbf{R e l}^{n} \mathbf{C a t}$ and objects $X, Y \in \boldsymbol{C}$, one can form a $\boldsymbol{T}$-diagram of arrow categories

$$
\boldsymbol{C}^{(\boldsymbol{T})}(X, Y): \boldsymbol{T} \longrightarrow \operatorname{Rel}^{n} \mathbf{C a t}
$$

which assigns to every object $T \in \boldsymbol{T}$ the arrow category

$$
C^{T}(X, Y) \in \mathbf{R e l}^{n} \mathbf{C a t}
$$

and to every map $t: T \rightarrow T^{\prime} \in \boldsymbol{T}$ the map

$$
t_{*}: \boldsymbol{C}^{T}(X, Y) \longrightarrow \boldsymbol{C}^{T^{\prime}}(X, Y) \in \operatorname{Rel}^{n} \mathbf{C a t}
$$

which sends a zigzag of type $T$

$$
X \stackrel{f_{1}}{X} \cdot \ldots \cdot \frac{f_{m}}{} Y
$$

to the zigzag of type $T^{\prime}$

$$
X \stackrel{f_{1}^{\prime}}{X} \cdot \ldots \cdot \frac{f_{m}^{\prime}}{Y} Y
$$

in which each $f_{j}^{\prime}\left(1 \leqslant j \leqslant m^{\prime}\right)$ is the composition of the $f_{i}$ with $t i=j$ or, in no such $i$ exists, the appropriate identity map.

Now we can form 


\subsection{The Grothendieck construction on $\boldsymbol{C}^{(\boldsymbol{T})}(X, Y)$}

Given an object $\boldsymbol{C} \in \mathbf{R e l}^{n+1}$ Cat and objects $X, Y \in \boldsymbol{C}$ the Grothendieck construction on $\boldsymbol{C}^{(\boldsymbol{T})}(X, Y)$ is the object

$$
\operatorname{Gr} \boldsymbol{C}^{(\boldsymbol{T})}(X, Y) \in \mathbf{R e l}^{n} \mathbf{C a t}
$$

which has

(i) as objects the zigzags in $C$ between $X$ and $Y$ in which the backward maps are in $\bar{a} \boldsymbol{C}$, i.e., pairs $(T, Z)$ consisting of objects

$$
T \in \boldsymbol{T} \quad \text { and } \quad Z \in \boldsymbol{C}^{T}(X, Y)
$$

and

(ii) for every two such objects $(T, Z)$ and $\left(T^{\prime}, Z^{\prime}\right)$, as maps $(T, Z) \rightarrow\left(T^{\prime}, Z^{\prime}\right)$ the pairs $(t, z)$ consisting of maps

$$
t: T \longrightarrow T^{\prime} \in \boldsymbol{T} \quad \text { and } \quad z: t_{*} Z \longrightarrow Z^{\prime} \in \boldsymbol{C}^{T^{\prime}}(X, Y)
$$

and in which

(iii) for every two composable maps $(t, z)$ and $\left(t^{\prime}, z^{\prime}\right)$ their composition is defined by the formula

$$
\left(t^{\prime}, z^{\prime}\right)(t, z)=\left(t^{\prime} t, z^{\prime}\left(t_{*} z\right)\right)
$$

Together these Grothendieck constructions give rise to

\subsection{A Grothendieck enrichment}

Given an object $\boldsymbol{C} \in \mathbf{R e l}^{n+1} \mathbf{C a t}$ we now define its Grothendieck enrichment as the category $\operatorname{Gr} \boldsymbol{C}^{(\boldsymbol{T})}$ enriched over $\mathbf{R e l}^{n} \mathbf{C a t}$ which

(i) has the same objects as $\boldsymbol{C}$,

(ii) has for every two objects $X, Y \in C$, as it's hom-object the $n$-relative category $\boldsymbol{C}^{(\boldsymbol{T})}(X, Y)$, and

(iii) has, for every three objects $X, Y$ and $Z \in C$ as composition

$$
\operatorname{Gr} \boldsymbol{C}^{(\boldsymbol{T})}(X, Y) \times \operatorname{Gr} \boldsymbol{C}^{(\boldsymbol{T})}(X, Y) \longrightarrow \operatorname{Gr} \boldsymbol{C}^{(\boldsymbol{T})}(X, Z)
$$

the function induced by the compositions of the zigzags involved.

\section{References}

[B] C. Barwick, Extended research statement, http://www.math.ias.edu/ clarkbar.

[BK1] C. Barwick and D. M. Kan, Relative categories: another model for the homotopy theory of homotopy theories, Indag. Math. (N.S.) 23 (2012), no. 1-2, $42-68$.

[DHKS] W. G. Dwyer, P. S. Hirschhorn, D. M. Kan, and J. H. Smith, Homotopy limit functors on model categories and homotopical categories, Mathematical Surveys and Monographs, vol. 113, American Mathematical Society, Providence, RI, 2004. 
[H] P. S. Hirschhorn, Model categories and their localizations, Mathematical Surveys and Monographs, vol. 99, American Mathematical Society, Providence, RI, 2003.

[La] D. M. Latch, The uniqueness of homology for the category of small categories, J. Pure Appl. Algebra 9 (1976/77), no. 2, 221-237.

[Lu] J. Lurie, $(\infty, 2)$-categories and the Goodwillie calculus I, available at http: //arxiv.org/abs/0905.0462.

[R] C. Rezk, A model for the homotopy theory of homotopy theory, Trans. Amer. Math. Soc. 353 (2001), no. 3, 973-1007 (electronic).

[T] R. W. Thomason, Cat as a closed model category, Cahiers Topologie Géom. Différentielle 21 (1980), no. 3, 305-324.

C. Barwick clarkbar@math.mit.edu

Department of Mathematics, Massachusetts Institute of Technology, Cambridge, MA 02139, USA

D. M. Kan (Deceased)

Department of Mathematics, Massachusetts Institute of Technology, Cambridge, MA 02139, USA 